\title{
Clinical Utility of Carotid Ultrasonography in the Prediction of Cardiovascular Events in Patients with Diabetes: A Combined Analysis of Data Obtained in Five Longitudinal Studies
}

\author{
Naoto Katakami ${ }^{1,2}$, Tomoya Mita ${ }^{3,4}$, Masahiko Gosho, ${ }^{5}$, Mitsuyoshi Takahara ${ }^{1,6}$, Yoko Irie ${ }^{7}$, \\ Tetsuyuki Yasuda ${ }^{7}$, Taka-aki Matsuoka ${ }^{1}$, Takeshi Osonoi ${ }^{8}$, Hirotaka Watada ${ }^{3,4,9}$ and lichiro Shimomura ${ }^{1}$ \\ ${ }^{1}$ Department of Metabolic Medicine, Osaka University Graduate School of Medicine, Osaka, Japan. \\ ${ }^{2}$ Department of Metabolism and Atherosclerosis, Osaka University Graduate School of Medicine, Osaka, Japan. \\ ${ }^{3}$ Department of Metabolism \& Endocrinology, Juntendo University Graduate School of Medicine, Tokyo, Japan. \\ ${ }^{4}$ Center for Molecular Diabetology, Juntendo University Graduate School of Medicine, Tokyo, Japan. \\ ${ }^{5}$ Department of Clinical Trial and Clinical Epidemiology, Faculty of Medicine, University of Tsukuba, Ibaraki, Japan. \\ ${ }^{6}$ Department of Diabetes Care Medicine, Osaka University Graduate School of Medicine, Osaka, Japan. \\ ${ }^{7}$ Osaka Police Hospital, Osaka, Japan. \\ ${ }^{8}$ Nakakinen Clinic, Ibaraki, Japan. \\ ${ }^{9}$ Center for Therapeutic Innovations in Diabetes, Juntendo University Graduate School of Medicine, Tokyo, Japan.
}

Aim: It remains unclear whether measures used in carotid ultrasonography such as the intima-media thickness (IMT) and ultrasonic tissue characterization of the carotid using the gray-scale median (GSM) can add prognostic information beyond the conventional cardiovascular risk markers in patients with diabetes.

Methods: This study employed a combined analysis of data obtained in five longitudinal studies including a total of 3263 patients with diabetes but without apparent cardiovascular disease (CVD) at baseline. The associations between carotid ultrasonography measures and the first occurrence of CVD (488 cases), which were defined as cardiovascular death, coronary artery diseases, stroke, or peripheral artery disease, were analyzed.

Results: Common carotid artery (CCA)-mean-IMT, CCA-max-IMT, Max-IMT, plaque-GSM, and the presence of low-GSM echolucent plaques at baseline were prognostic factors for CVD even after adjustment for conventional risk factors. Time-dependent receiver-operating-characteristic (ROC) curve analysis indicated that the use of CCA-mean-IMT, CCA-max-IMT, and Max-IMT in addition to the conventional risk factors improved significantly the prediction of occurrence of CVD. Increments in the CCA-mean-IMT (hazard ratio [HR] 2.37 for every $0.1-\mathrm{mm} /$ year increment [95\% confidence interval [CI]: 1.63-3.47], $p<0.001$ ), Max-IMT (HR 1.51 for every $0.1-\mathrm{mm} /$ year increment [95\% CI: $1.07-2.14$ ],$p=0.020$ ), and Mean-GSM (HR 0.22 for every 10-U/year increment [95\% CI: 0.06-0.76], $p=0.016$ ) during the observation period were also prognostic factors for CVD even after adjusting for the baseline value of the respective measure.

Conclusions: Addition of carotid ultrasonography measures to conventional risk factors significantly improved the stratification of patients by cardiovascular risk. Changes over time in carotid ultrasonography measures may be used as therapeutic outcome measures.

Key words: IMT, Carotid plaque, Tissue characterization, Cardiovascular diseases, Diabetes mellitus

Abbreviations: CAC, coronary artery calcium; CCA, common carotid artery; CVD, cardiovascular disease; DM, diabetes mellitus; FRS, Framingham Risk Score; GSM, gray-scale median; HR, hazard ratio; IMT, intima-media thickness; $\mathrm{PAD}$, peripheral artery disease

Address for correspondence: Naoto Katakami, Department of Metabolic Medicine, Osaka University Graduate School of Medicine, 2-2, Yamadaoka, Suita, Osaka 565-0871, Japan. E-mail: katakami@endmet.med.osaka-u.ac.jp

Received: October 30, 2017 Accepted for publication: December 21, 2017

Copyright@2018 Japan Atherosclerosis Society

This article is distributed under the terms of the latest version of CC BY-NC-SA defined by the Creative Commons Attribution License. 


\section{Introduction and Aim}

Carotid ultrasonography is a simple and noninvasive procedure that has allowed clinicians to visualize the characteristics of the carotid wall and lumen surfaces to quantify the severity of atherosclerosis ${ }^{1}$. In particular, carotid intima-media thickness (IMT) is related to multiple conventional risk factors ${ }^{1-3)}$ and can be a simple and useful marker of subclinical arterial damage, an index located somewhere between risk factors and "hard" clinical endpoint events such as myocardial infarction and stroke. IMT has indeed been shown to be a predictor of cardiovascular disease $(\mathrm{CVD})^{1,4-11}$. However, several previous studies indicated that the addition of IMT to conventional risk factors could bring about at most just a small improvement in the ability to predict CVD in the general population ${ }^{1,12-15)}$. In addition, if IMT was a valid surrogate for CVD events, both a single measurement of IMT and changes in IMT over time (IMT change) could be independent predictors of future clinical events. However, recent meta-analyses cast doubt on the validity of IMT change as a surrogate marker ${ }^{16,177}$.

Recent studies have revealed that ultrasonic tissue characterization of carotid plaques using integrated backscatter and the gray-scale median (GSM) can also be an indicator of cardiovascular events ${ }^{18-22}$.

However, it has not been determined whether carotid ultrasonographic measures, including IMT and GSM, can add prognostic information beyond the conventional risk factors in patients with diabetes mellitus (DM).

In this context, the present study investigated (1) whether carotid IMT or GSM are prognostic factors for CVD after adjusting for conventional risk factors; (2) in case they are, whether the use of IMT or GSM in addition to conventional risk factors or an established cardiovascular risk score based on these can better stratify patients into cardiovascular risk groups; and (3) whether change (regression or progression) over time in IMT or GSM can be used as a therapeutic outcome measure, based on statistical analyses.

\section{Methods}

Subjects

This study employed a combined analysis of data obtained in five longitudinal studies in Japanese patients with DM (based on the criteria of the Japan Diabetes Society) who were followed-up for the occurrence of CVD as outcome measures. The list of the five longitudinal studies from which data were utilized to construct the combined dataset is as follows:
(1) "The development of tailor-made treatment for diabetic complications using DNA chips," an observational study that was conducted at the Osaka University Hospital and Naka-Kinen Hospital from January 11, 2005 to March 24, 2015.

(2) "Predictive value of carotid intima-media thickness for cardiovascular disease in type 2 diabetic patients," an ongoing observational study that started in 2003 at the Juntendo University Hospital ${ }^{23)}$.

(3) "Clinical research on identifying patients at high risk for severe coronary disease among those with asymptomatic type 2 diabetes mellitus without a history of coronary artery disease," an ongoing observational study that started in 2007 at the Osaka Police Hospital.

(4) SPEAD-A Study, a clinical study started at the Juntendo University Hospital and other institutions in 2011 24, 25), and its extension study called "SPEAD-A Extension Study."

(5) SPIKE Study, a clinical study started at the Juntendo University Hospital and other institutions in $2012^{26,27)}$, and its extension study called the "SPIKE Extension Study."

The aim and the eligibility criteria of these studies are shown in Supplemental Table 1.

The study subjects of the present study were from combined data according to the following eligibility criteria:

Inclusion criteria: Patients who underwent carotid ultrasonography at baseline.

Exclusion criteria: 1) Patients with a history of CVD at baseline; 2) patients who declined to participate in this study.

The study protocol was approved by the ethics committees for human research of the Osaka University Hospital and the study was conducted in accordance with the principles of the Helsinki Declaration. This study has been registered on the University Hospital Medical Information Network Clinical Trials Registry, which is a non-profit organization in Japan and meets the requirements of the International Committee of Medical Journal Editors (UMIN000020108).

\section{Outcome Measures}

The primary outcome measure was the first occurrence of CVD events. In the present study, CVD events were defined as cardiovascular death, coronary artery disease (i.e., acute myocardial infarction, angina pectoris, or revascularization for the treatment of coronary artery diseases), stroke (ischemic or hemorrhagic stroke), or peripheral artery disease (PAD).

Diagnosis of occurrence of coronary artery disease was performed by cardiologists based on the clini- 
cal symptoms, characteristic ECG changes, cardiac enzyme levels, and findings on coronary angiography and/or echocardiography according to established guidelines ${ }^{28,29)}$. An ischemic stroke event was defined as a validated, definite or probable hospitalized atherothrombotic, cardioembolic, lacunar, or other type of ischemic stroke, diagnosed by neurological experts based on clinical symptoms and neuroimaging findings, according to the National Institute of Neurological Disorders and Stroke (NINDS) III classification ${ }^{30)}$. The presence of lower extremity PAD was defined as occlusion or severe stenosis observed on either the right or left side. Subjects whose ABI value decreased to below 0.9 were diagnosed as having developed PAD.

For participants with a CVD event, follow-up duration was defined as the period between the baseline clinic visit and the date of the first CVD event. For participants with no CVD event, follow-up continued until the date of death or March 31, 2016, or until the date of last contact.

\section{Factors Related to Carotid Ultrasonography}

In the assessment of measures of carotid ultrasonography, a unified method was used in the abovementioned five studies. Scans of the carotid artery were performed by expert sonographers based on the guideline of the Japan Society of Ultrasonics in Medicine $^{31)}$, using high-resolution B-mode ultrasound scanners equipped with a high frequency $(>7.5-\mathrm{MHz})$ linear transducer, with a limit of detection of $<0.1$ $\mathrm{mm}$. The carotid arteries were scanned in transverse sections from the origins of the common carotid arteries (CCA), carotid sinuses, internal carotid arteries (ICA), and external carotid arteries (ECA), and then examined for carotid lesions in longitudinal sections at different angles (anterior, lateral, and posterior, which approximately correspond to 60,90, and 150 degrees for the right carotid artery, and 210, 270, and 300 degrees for the left carotid artery marked on Meijer's Arc). The site of greatest thickness, including plaque lesions, was then sought along the arterial walls.

The IMT is defined as the distance between two parallel echogenic lines corresponding to the vascular lumen and the adventitial layer. In this study, localized elevated lesions with a maximum thickness of $>1$ $\mathrm{mm}$, having a point of inflection on the surface of the intima-media complex, are defined as "carotid plaque" based on the guideline of the Japan Society of Ultrasonics in Medicine ${ }^{31)}$. It should be also noted that the measurement of IMT was performed at the site of greatest thickness, "including plaque lesions," in accordance with the recommendation of the Japan Society of Ultrasonics in Medicine ${ }^{31)}$. CCA-mean-
IMT was calculated using an automatic IMT-measuring software installed on ultrasound scanners.

The echogenicity of the arterial wall was evaluated based on the GSM method in a gray-scale range of 0 to 255 ( 0 as the darkest tone and 255 as the brightest). Adobe Photoshop software, version 7.0 (Adobe Systems, San Jose, CA, USA) was used for image standardization and calculation of gray-scale values. In accordance with the previous report, the standardization of the B-mode image was performed using a curve option, so that the GSM for the blood ranged from $0-5$, and for the adventitia from 185 195 ${ }^{32)}$. Then, the Mean-IMT-CCA area (the segment $2 \mathrm{~cm}$ proximal to the dilation of the carotid bulb) was delineated with a freehand tool, and the GSM of the selected area was read from the entire delineated area. Similarly, if there was an atherosclerotic plaque lesion (i.e., focal elevated lesion with Max-IMT-CCA > $1.0 \mathrm{~mm}$ ), the GSM of the plaque lesion was also measured using the same method. In the event that multiple plaque lesions were found in one individual, the plaque with the greatest thickness was subject to GSM measurement and the GSM value was used as the subject's representative value.

The following five measures were analyzed at the carotid ultrasonography in the present study:

- CCA-mean-IMT: The mean common carotid IMT was determined in the right and left carotid arteries, and the larger of the two values was used.

- CCA-max-IMT: The maximum common carotid IMT was determined in the right and left carotid arteries, and the larger of the two values was used.

- Max-IMT: The maximum IMT in the segment from the CCA to the ICA was determined in the right and left carotid arteries, and the larger of the two values was used.

- Mean-GSM: The GSM of the IMT was determined at the segment $2 \mathrm{~cm}$ proximal to the dilation of the carotid bulb in the right and left carotid arteries, and the mean of the two values was used.

- Plaque-GSM: The GSM value of carotid plaque lesions (Max-IMT $>1.0 \mathrm{~mm}$ ) was determined in the right and left carotid arteries, and the smaller of the two values was used.

According to these definitions, the numbers of subjects whose data of such IMT measures were available from original studies were as follows: 3260 patients for CCA-mean-IMT, 2243 patients for CCAmax-IMT, 540 patients for Max-IMT, 529 patients for Mean-GSM, and 534 patients for plaque-GSM.

We do not have comprehensive quality control data of IMT measurements since this study employed an integrated data analysis obtained in five indepen- 
dent studies. However, the reproducibility of the IMT measurements in each study was quite high (the mean absolute difference between two measurements ranged from $0.02 \pm 0.01$ to $0.05 \pm 0.07 \mathrm{~mm}$ ). Regarding GSM, the intra- and inter-reader coefficients of variation for GSM measurements was $2.9 \%-4.8 \%$ and $8.2 \%$, respectively, for 40 consecutively replicated measurements, demonstrating high reproducibility.

Conventional Risk Factors for CVD Events (as Adjustment Factors)

In the present study, conventional risk factors for CVD were defined as sex, age, cigarette smoking, hypertension, dyslipidemia, obesity, HbA1c levels, duration of DM, and higher serum creatinine value, on the basis of previous studies. These clinical and biochemical data were collected and measured using standard laboratory protocols at baseline in each study. The Framingham Risk Score (FRS), calculated with the Framingham-D'Agostino risk equation, was used as a typical cardiovascular disease risk score based on conventional risk factors such as sex, age, total cholesterol, HDL cholesterol, systolic blood pressure (SBP), anti-hypertensive medication use, current smoking, and DM status ${ }^{33)}$. The determination of hypertension (defined as $\mathrm{SBP} \geq 130 \mathrm{mmHg}$ or diastolic blood pressure $\geq 80 \mathrm{mmHg}$ or anti-hypertensive medication use) and dyslipidemia (defined as serum LDL cholesterol $\geq$ $3.1 \mathrm{mmol} / \mathrm{L}[120 \mathrm{mg} / \mathrm{dL}]$ or serum TG $\geq 1.7 \mathrm{mmol} / \mathrm{L}$ $[150 \mathrm{mg} / \mathrm{dL}]$ or HDL-cholesterol $<1.0 \mathrm{mmol} / \mathrm{L}$ [ 40 $\mathrm{mg} / \mathrm{dL}]$ or use of lipid-lowering medication) was based on the criteria of the Japan Diabetes Society ${ }^{34)}$. The determination of obesity (defined as body mass index $[\mathrm{BMI}] \geq 25.0 \mathrm{~kg} / \mathrm{m}^{2}$ ) was based on the Japan Society for the Study of Obesity's criteria. The individuals who currently smoked at least one cigarette per day were defined as current smokers.

\section{Statistical Analysis}

All values are reported as mean \pm SD for continuous variables, or percentage in parentheses for categorical variables. Occurrence of the above-mentioned CVD events was plotted using the Kaplan-Meier method and differences between the groups were assessed by a log-rank test. The occurrence of the CVD events was analyzed using the Cox proportional hazards model including each carotid ultrasonography measure, conventional risk factors (or FRS), and the original study in which each subject participated as explanatory variables to estimate hazard ratios (HRs) and $95 \%$ confidence intervals (CIs).

The ability of variables to predict CVD outcome was examined by time-dependent receiver-operatingcharacteristic (ROC) curve analysis. In order to inves- tigate whether the addition of IMT or GSM to the conventional risk factors improves the stratification of patients by CVD event risk, the C-statistics were calculated ${ }^{35)}$. The Youden index, that is, the sum of the sensitivity and specificity minus one, was used to determine a cut-off value for each carotid ultrasonography measure. The net reclassification improvement (NRI) statistics were calculated by categorizing the 10 -year event rate into $<10 \%, 10 \%-20 \%$, and $\geq$ $20 \%{ }^{36)}$.

The following methods were used to investigate whether change over time in each carotid ultrasonography measure can be used as a measure of therapeutic response. A univariate regression analysis was conducted for individual patients to estimate the slope of each carotid ultrasonography measures over time. For patients who experienced CVD events during the observation period, data before the occurrence of the first event were used to calculate the slope. Data after the first event were excluded because changes in IMT or GSM may be significantly affected by the event and including post-event data to calculate the slope is clinically considered inappropriate, as well as because IMT or GSM may often not be determined after the occurrence of CVD events. Data at all time points were used for patients who did not experience CVD events during the observation period to improve the precision of the slope estimate. Next, a Cox proportionalhazards model was developed using the occurrence of the above-mentioned CVD events as the response variable, the baseline value of each carotid ultrasonography measure, the slope obtained as described above, and the conventional risk factors or FRS as explanatory variables.

The above analyses were conducted without imputation for missing data, and cases with missing data were excluded from the analysis. For all tests, $p<$ 0.05 was considered statistically significant. The statistical analyses were performed using SAS 9.3 (SAS Institute, Cary, NC, USA).

\section{Results}

Among 3945 patients registered in the database, 3263 patients who met the selection criteria were included in the analysis set for the present study, with males accounting for $65.5 \%$ and a mean age of $60.9 \pm$ 9.9 years. Table 1 summarizes the clinical characteristics of the patients at baseline. During the observation period ( $81 \pm 41$ months), CVD events developed in 488 patients and major adverse cardiovascular events (MACE) developed in 295 patients. Coronary artery disease developed in 240 patients. Stroke developed in 257 patients and PAD developed in 50 patients. Fifty 
Table 1. Baseline characteristics of study subjects

\begin{tabular}{lcccc}
\hline & $\begin{array}{c}\text { Entire sample } \\
(n=3263)\end{array}$ & $\begin{array}{c}\text { Without event } \\
(n=2775)\end{array}$ & $\begin{array}{c}\text { With event } \\
(n=488)\end{array}$ & $p$ value \\
Parameters & 65.5 & 64.2 & 73.0 & $<0.001$ \\
Gender (male, \%) & $60.9 \pm 9.7$ & $60.3 \pm 10.1$ & $64.5 \pm 7.8$ & $<0.001$ \\
Age (years) & 39.9 & 38.4 & 48.4 & $<0.001$ \\
Current smoker (\%) & $8.4 \pm 7.9$ & $8.5 \pm 7.9$ & $7.8 \pm 7.6$ & 0.07 \\
Duration of diabetes (years) & $7.5 \pm 1.3$ & $7.5 \pm 1.3$ & $7.5 \pm 1.3$ & 0.38 \\
HbAlc (\%) & 35.1 & 35.7 & 32.0 & 0.12 \\
Obesity (\%) & 57.3 & 54.9 & 70.7 & $<0.001$ \\
Hypertension (\%) & 64.4 & 64.6 & 63.7 & 0.72 \\
Dyslipidemia (\%) & $0.8 \pm 0.5$ & $0.8 \pm 0.4$ & $0.9 \pm 0.9$ & $<0.001$ \\
Serum creatinine (mg/dl) & $31.3 \pm 19.1$ & $29.8 \pm 18.7$ & $39.2 \pm 19.5$ & $<0.001$ \\
Framingham risk (10-year CVD, \%) & & & & 0.14 \\
Administration of & 83.6 & 83.2 & 85.9 & $<0.001$ \\
$\quad$ Anti-diabetic drugs (\%) & 43.5 & 42.0 & 52.5 & 0.21 \\
$\quad$ Anti-hypertensive drugs (\%) & 37.6 & 37.2 & 40.2 & $<0.001$ \\
Anti-hyperlipidemic drugs (\%) & $0.9 \pm 0.3$ & $0.9 \pm 0.3$ & $1.0 \pm 0.4$ & $<0.001$ \\
CCA-mean-IMT (mm) ( $n=3260)$ & $1.1 \pm 0.4$ & $1.1 \pm 0.3$ & $1.2 \pm 0.4$ & $<0.001$ \\
CCA-max-IMT (mm) $(n=2243)$ & $1.8 \pm 0.9$ & $1.7 \pm 0.8$ & $2.4 \pm 1.0$ & 0.85 \\
Max-IMT (mm) $(n=540)$ & $55.5 \pm 15.8$ & $55.5 \pm 15.9$ & $56.4 \pm 11.5$ & $<0.001$ \\
Mean-GSM (U) $(n=529)$ & $50.4 \pm 17.8$ & $51.3 \pm 17.7$ & $42.0 \pm 16.5$ & \\
Plaque-GSM $(\mathrm{U})(n=534)$ & & & \\
\hline
\end{tabular}

Data are shown as $\%$ or means \pm SD. $P$ values were calculated by Student's $t$-test or chi-square as appropriate. Abbreviations: IMT, intima-media thickness; GSM, gray-scale median.

Individuals who currently smoked at least one cigarette per day were defined as current smokers. Obesity was defined as body mass index $\geq 25.0 \mathrm{~kg} / \mathrm{m}^{2}$ ). Hypertension was defined as systolic blood pressure $\geq 130 \mathrm{mmHg}$ or diastolic blood pressure $\geq 80 \mathrm{mmHg}$ or anti-hypertensive medication use. Dyslipidemia was defined as serum LDL cholesterol $\geq 3.1$ $\mathrm{mmol} / \mathrm{L}(120 \mathrm{mg} / \mathrm{dl})$ or serum TG $\geq 1.7 \mathrm{mmol} / \mathrm{L}(150 \mathrm{mg} / \mathrm{dl})$ or HDL-cholesterol $<1.0 \mathrm{mmol} / \mathrm{L}(40 \mathrm{mg} / \mathrm{dl})$ or use of lipid-lowering medication.

patients died.

(1) Carotid IMT and GSM are Prognostic Factors for the Occurrence of CVD Events even after Adjusting for Conventional Risk Factors

Data used to analyze the predictive performance of carotid ultrasonography measures refer to the following numbers of patients: 3260 patients for CCAmean-IMT, 2243 patients for CCA-max-IMT, 540 patients for Max-IMT, 529 patients for Mean-GSM, and 534 patients for plaque-GSM (Table 1).

When evaluable patients were divided by quintile for each carotid ultrasonography measure, to assess the relationship between the measure and the occurrence of CVD events, the risk of CVD events was higher in those with higher CCA-mean-IMT, CCA-max-IMT, or Max-IMT (log-rank test, $p<0.001$ for all measures; Fig. 1A, 1B, 1C). Although no significant relationship was observed between mean-GSM and the occurrence of CVD events (Fig. 1D), the risk of CVD events was significantly higher in the first quintile of patients with the lowest plaque-GSM than in the four higher quintiles (log-rank test, $p<0.001$ ) (Fig. 1E).

Multivariate Cox regression analyses revealed that CCA-mean-IMT (HR 1.08 for every $0.1-\mathrm{mm}$ increment [95\% CI: 1.05-1.11], $p<0.001)$, CCA-maxIMT (HR 1.07 for every $0.1-\mathrm{mm}$ increment [95\% CI: 1.04-1.10], $p<0.001$ ), Max-IMT (HR 1.08 for every $0.1-\mathrm{mm}$ increment [95\% CI: $1.05-1.11], p<0.001)$, plaque-GSM (HR 0.74 for every $10-\mathrm{U}$ increment [95\% CI: $0.62-0.90], p=0.002)$, and the presence of low-GSM ( $\leq 35)$ echolucent plaque (HR $2.90[95 \%$ CI: 1.60-5.24], $p<0.001)$ were prognostic factors for CVD events even after adjustment for the original study in which each subject participated and nine conventional risk factors (sex, age, smoking, hypertension, dyslipidemia, obesity, HbA1c, duration of DM, and serum Cr level) (Table 2, model 2). Similarly, after adjustment for the study variable and FRS, CCA-mean-IMT, CCA-max-IMT, Max-IMT, plaqueGSM, and the presence of low-GSM plaques were prognostic factors for CVD (Table 2, model 3). 


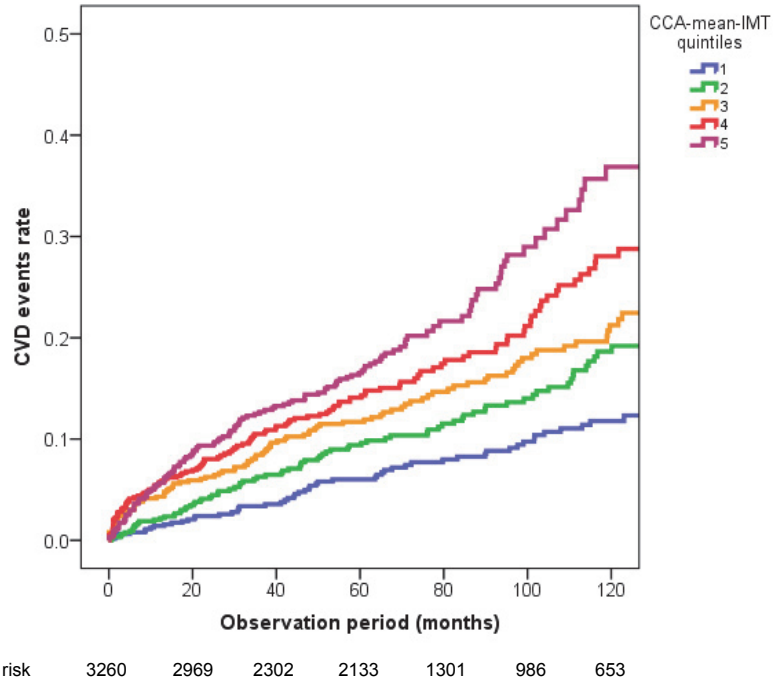

Fig. 1A. Kaplan-Meier curves depicting the cumulative probability of cardiovascular events in Japanese patients with type $2 \mathrm{DM}$

A total of 3260 subjects were divided into five subgroups by quintiles according to their baseline CCA-mean-IMT values (Q1, $\leq$ $0.72 \mathrm{~mm}$; Q2, 0.73-0.82 mm; Q3, 0.83-0.92 mm; Q4, 0.93$1.09 \mathrm{~mm}$; and Q5, $\geq 1.10 \mathrm{~mm}$ ) and followed-up to assess the relationship between the baseline CCA-mean-IMT and the occurrence of cardiovascular events. The risk for cardiovascular events was higher in patients with increased baseline CCA-mean-IMT (logrank test, $p<0.001$ ).

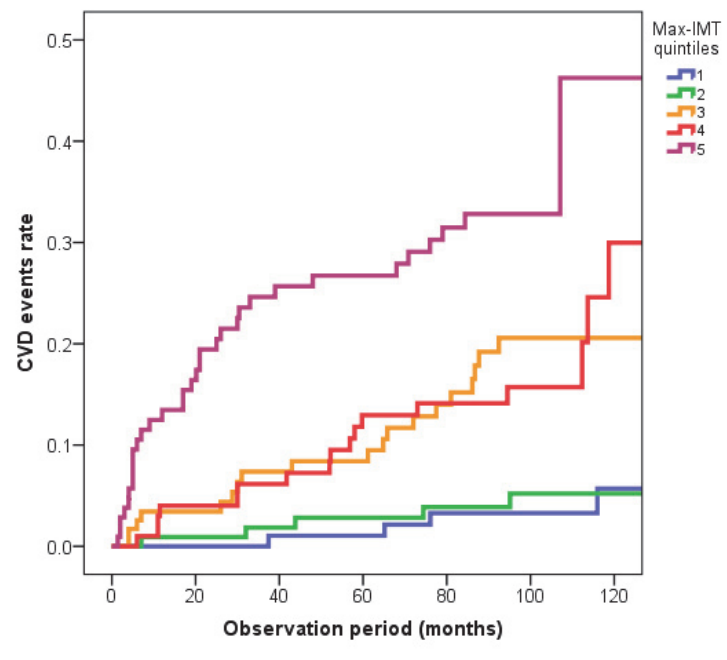

$\begin{array}{llllllll}\text { No. at risk } & 540 & 485 & 445 & 417 & 368 & 166 & 96\end{array}$

Fig. 1C. Kaplan-Meier curves depicting the cumulative probability of cardiovascular events in Japanese patients with type $2 \mathrm{DM}$

A total of 540 subjects were divided into five subgroups by quintiles according to their baseline Max-IMT values (Q1, $\leq 1.05 \mathrm{~mm}$; Q2, 1.06-1.38 mm; Q3, 1.39-1.90 mm; Q4, 1.91-2.57 mm; and $\mathrm{Q} 5, \geq 2.58 \mathrm{~mm}$ ) and followed-up to assess the relationship between the baseline Max-IMT and the occurrence of cardiovascular events. The risk for cardiovascular events was higher in patients with increased baseline Max-IMT (log-rank test, $p<0.001$ ).

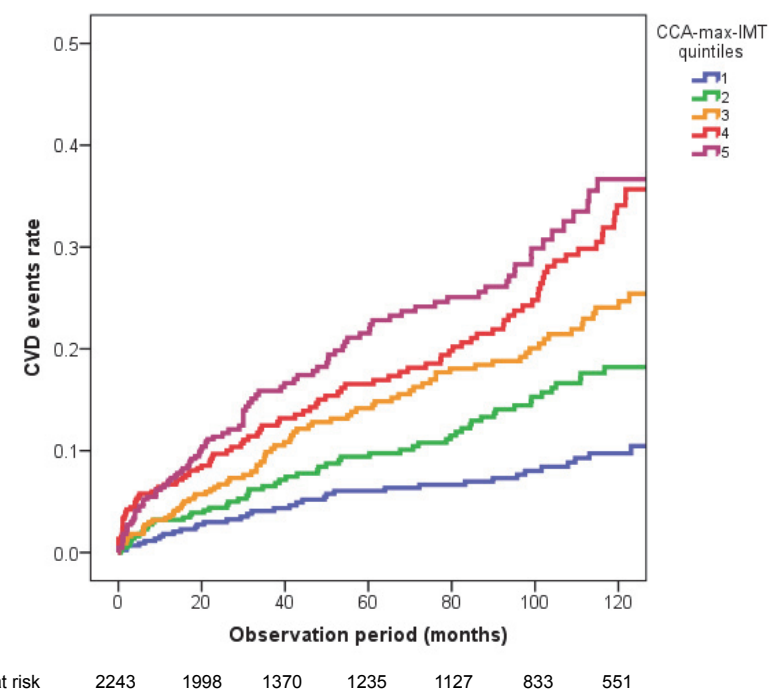

Fig. 1B. Kaplan-Meier curves depicting the cumulative probability of cardiovascular events in Japanese patients with type $2 \mathrm{DM}$

A total of 2243 subjects were divided into five subgroups by quintiles according to their baseline CCA-max-IMT values $(\mathrm{Q} 1, \leq 0.84$ $\mathrm{mm}$; Q2, 0.85-0.95 mm; Q3, 0.96-1.08 mm; Q4, 1.09-1.25 $\mathrm{mm}$; and $\mathrm{Q} 5, \geq 1.26 \mathrm{~mm}$ ) and followed-up to assess the relationship between the baseline CCA-max-IMT and the occurrence of cardiovascular events. The risk for cardiovascular events was higher in patients with increased baseline CCA-max-IMT (log-rank test, $p<0.001)$.

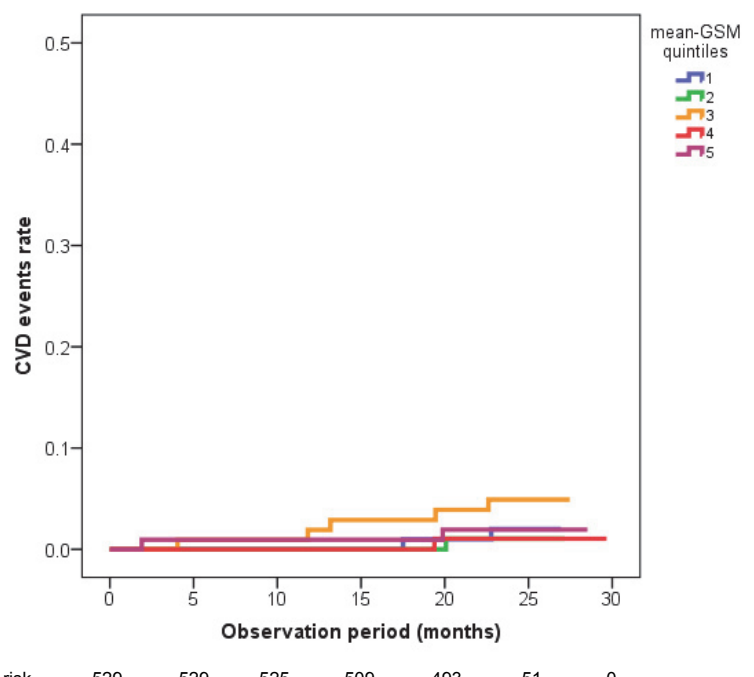

$\begin{array}{llllllll}\text { No. at risk } & 529 & 529 & 525 & 509 & 493 & 51 & 0\end{array}$

Fig. 1D. Kaplan-Meier curves depicting the cumulative probability of cardiovascular events in Japanese patients with type $2 \mathrm{DM}$

A total of 529 subjects were divided into five subgroups by quintiles according to their baseline Mean-GSM values (Q1, $\leq 42.0 \mathrm{U}$; Q2, 42.5-49.5 U; Q3, 50.0-58.5 U; Q4, 59.0-67.5 U; and Q5, $\geq$ $68.0 \mathrm{U})$ and followed-up to assess the relationship between the baseline Mean-GSM and the occurrence of cardiovascular events. No significant relationship was observed between the Mean-GSM and the occurrence of cardiovascular events. 
(2) Carotid Ultrasonography Measures Used in Addition to Conventional Risk Factors can Better Stratify Patients into Cardiovascular Risk Groups

The time-dependent ROC curve analysis indicated that a predictive model using the above-mentioned nine conventional risk factors is useful in predicting the occurrence of CVD events (C-statistic 0.699; $p<0.001)$. On the other hand, the use of

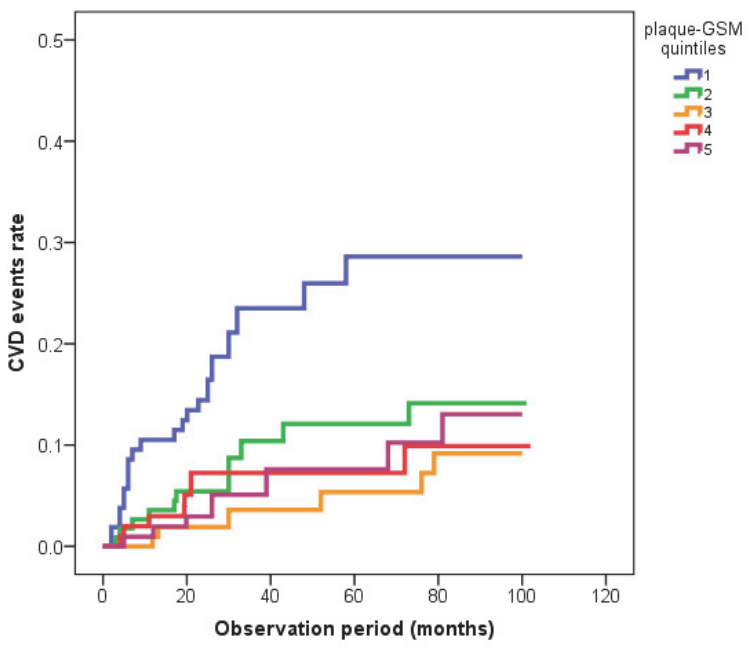

$\begin{array}{llllllll}\text { No. at risk } & 534 & 474 & 218 & 201 & 176 & 10 & 0\end{array}$

Fig. 1E. Kaplan-Meier curves depicting the cumulative probability of cardiovascular events in Japanese patients with type $2 \mathrm{DM}$

A total of 534 subjects were divided into five subgroups by quintiles according to their baseline plaque-GSM values $(\mathrm{Q} 1, \leq 35 \mathrm{U}$;

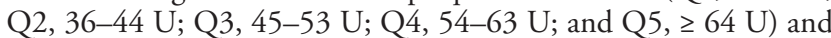
followed-up to assess the relationship between the baseline plaqueGSM and the occurrence of cardiovascular events. The risk of cerebro-/cardiovascular events was significantly higher in the first quintile of patients with the lowest plaque-GSM than in the four higher quintiles (log-rank test, $p<0.001$ ).
CCA-mean-IMT, CCA-max-IMT, or Max-IMT in addition to the nine conventional risk factors significantly improved the prediction of occurrence of CVD events (delta C-statistic 0.009 [95\% CI: $0.002-$ $0.020], p=0.006$ for CCA-mean-IMT, delta C-statistic 0.014 [95\% CI: 0.002-0.027], $p=0.017$ for CCAmax-IMT, and delta C-statistic 0.093 [95\% CI: 0.017-0.170], $p=0.008$ for Max-IMT) (Table 3). The C-statistics did not increase when plaque-GSM or the presence of low-GSM plaques was added to the conventional risk factors (delta C-statistic - 0.037 [95\% CI: $-0.078-0.066$ ], $p=0.912$ for plaque-GSM; and delta C-statistic 0.007 [95\% CI: -0.026-0.088], $p=0.432$ for the presence of low-GSM plaque) (Table 3).

Although FRS alone is also useful in predicting the occurrence of CVD events (C-statistic, 0.655; $p<$ 0.001 ), adding CCA-max-IMT or Max-IMT to the FRS significantly improved the prediction of CVD events (delta C-statistics 0.019 [95\% CI: 0.006 $0.036], p=0.007$, and delta C-statistics $0.124[95 \%$ CI: 0.048-0.216], $p<0.001$, respectively) (Table 3). Similarly, the C-statistics tended to increase when CCA-mean-IMT was added to the FRS, although the change was not statistically significant (delta C-statistic 0.010 [95\% CI: $-0.001-0.025$ ], $p=0.064$ ).

The use of any carotid ultrasonography measures in addition to the classic risk factors did not increase NRI significantly.

(3) Changes over Time in IMT and GSM are Prognostic Factors for CVD Events

Data were available to analyze the associations between changes over time in carotid ultrasonography measures and onset of CVD in the following numbers of patients: 1881 patients for CCA-mean-IMT, 1741 patients for CCA-max-IMT, 133 patients for Max-

Table 2. Relative risk of cardiovascular events for ultrasonographic variables

\begin{tabular}{|c|c|c|c|c|c|c|}
\hline Parameters & $\begin{array}{c}\text { Model } 1 \\
\text { HR }(95 \% C I)\end{array}$ & $p$ value & $\begin{array}{c}\text { Model } 2 \\
\text { HR }(95 \% C I)\end{array}$ & $p$ value & $\begin{array}{c}\text { Model } 3 \\
\text { HR }(95 \% C I)\end{array}$ & $p$ value \\
\hline CCA-mean-IMT (per $0.1 \mathrm{~mm}$ ) & $1.12(1.10-1.15)$ & $<0.001$ & $1.08(1.05-1.11)$ & $<0.001$ & $1.10(1.07-1.12)$ & $<0.001$ \\
\hline Max-IMT (per $0.1 \mathrm{~mm})$ & $1.08(1.06-1.10)$ & $<0.001$ & $1.08(1.05-1.11)$ & $<0.001$ & $1.07(1.05-1.09)$ & $<0.001$ \\
\hline Mean-GSM (per $10 \mathrm{U})$ & $1.07(0.74-1.54)$ & 0.72 & $1.10(0.72-1.66)$ & 0.66 & $1.08(0.75-1.56)$ & 0.68 \\
\hline
\end{tabular}

Cox proportional hazards regression analyses adjusted for the following covariates:

Model 1, adjusted for the original study in which each subject participated.

Model 2, adjusted for the original study in which each subject participated, gender, age, current smoking, hypertension, dyslipidemia, obesity, HbA1c, duration of diabetes, and serum creatinine value.

Model 3, adjusted for the original study in which each subject participated and the Framingham risk score.

Abbreviations: HR, Hazard Ratio; IMT, intima-media thickness; GSM, gray-scale median. 
Table 3. Contribution of different carotid ultrasonography measures to conventional risk factors in the prediction of CVD

\begin{tabular}{|c|c|c|c|c|}
\hline Parameters & $\begin{array}{l}\text { vs. conventional risk factors alone } \\
\text { delta C-statistic }(95 \% \mathrm{CI})\end{array}$ & $p$ value & $\begin{array}{l}\text { vs. Framingham Risk Score alone } \\
\text { delta C-statistic }(95 \% \mathrm{CI})\end{array}$ & $p$ value \\
\hline CCA-mean-IMT & 0.009 (95\% CI: 0.002 to 0.020$)$ & 0.006 & 0.010 (95\% CI: -0.001 to 0.025$)$ & 0.064 \\
\hline Max-IMT & 0.093 (95\% CI: 0.017 to 0.170$)$ & 0.008 & 0.124 (95\% CI: 0.048 to 0.216$)$ & $<0.001$ \\
\hline Mean-GSM & 0.001 (95\% CI: -0.021 to 0.024$)$ & 0.986 & 0.008 (95\% CI: -0.026 to 0.091$)$ & 0.535 \\
\hline
\end{tabular}

Each carotid ultrasonography measure was combined with nine conventional risk factors (gender, age, current smoking, hypertension, dyslipidemia, obesity, HbA1c, duration of diabetes mellitus, and serum creatinine value) and the original study in which each subject participated or the Framingham Risk Score and the study. The increase of C-statistics (delta C-statistics) were calculated using time-dependent ROC curve analyses

Table 4. Relative risk of cardiovascular events with respect to changes over time (increment per year) in ultrasonographic variables

\begin{tabular}{|c|c|c|c|c|c|c|}
\hline Parameters & $\begin{array}{c}\text { Model } 1 \\
\text { HR }(95 \% C I)\end{array}$ & $p$ value & $\begin{array}{c}\text { Model } 2 \\
\text { HR }(95 \% \text { CI })\end{array}$ & $p$ value & $\begin{array}{c}\text { Model } 3 \\
\text { HR }(95 \% C I)\end{array}$ & $p$ value \\
\hline CCA-mean-IMT (per $0.1 \mathrm{~mm} /$ year) & $2.37(1.63-3.47)$ & $<0.001$ & $1.77(1.18-2.66)$ & 0.006 & $1.98(1.34-2.94)$ & $<0.001$ \\
\hline Max-IMT (per $0.1 \mathrm{~mm} /$ year) & $1.51(1.07-2.14)$ & 0.020 & $1.44(0.64-3.25)$ & $0.38^{\dagger}$ & $1.47(0.96-2.25)$ & 0.07 \\
\hline Mean-GSM (per 10 U/year) & $0.22(0.06-0.76)$ & 0.016 & $0.23(0.07-0.83)$ & $0.025^{\dagger}$ & $0.25(0.08-0.82)$ & 0.022 \\
\hline
\end{tabular}

Cox proportional hazards regression analyses adjusted for the following covariates:

Model 1, adjusted for baseline value and the original study in which each subject participated.

Model 2, adjusted for baseline value, the original study in which each subject participated, gender, age, current smoking, hypertension, dyslipidemia, obesity, HbA1c, duration of diabetes, and serum creatinine value on the basis of previous studies.

Model 3, adjusted for baseline value, the original study in which each subject participated, and the Framingham risk score

${ }^{\dagger}$ Validity of the analysis result is questionable because the number of exploratory variables is too much than the number of events.

IMT, 500 patients for Mean-GSM, and 227 patients for plaque-GSM. The increment in CCA-mean-IMT (HR 2.37 for every $0.1-\mathrm{mm} /$ year increment $[95 \% \mathrm{CI}$ : 1.63-3.47], $p<0.001$ ), increment in Max-IMT (HR 1.51 for every $0.1-\mathrm{mm} /$ year increment $[95 \% \mathrm{CI}$ : $1.07-2.14], p=0.020)$, and increment in Mean-GSM (HR 0.22 for every 10-U/year increment [95\% CI: $0.06-0.76], p=0.016$ ) were prognostic factors for CVD even after adjusting for the baseline value of the respective measure (Table 4). The risk of CVD event occurrence tended to be higher in patients with a larger increment in CCA-max-IMT, but the difference was not statistically significant (HR 1.20 for every $0.1-\mathrm{mm} /$ year increment [95\% CI: 0.94-1.53], $p=$ $0.15)$. Change in plaque-GSM did not significantly relate to CVD events risk. Furthermore, the increment in CCA-mean-IMT was a prognostic factor for CVD events even after adjusting for the conventional risk factors or FRS at baseline (HR 1.77 for every $0.1-\mathrm{mm} /$ year increment [95\% CI: 1.18-2.66], $p=0.006$; HR 1.98 for every $0.1-\mathrm{mm} /$ year increment $[95 \% \mathrm{CI}$ : 1.34-2.94], $p<0.001)$. Similarly, change in MeanGSM was also a prognostic factor for CVD events occurrence even after adjusting for the conventional risk factors or FRS (HR 0.23 for every 10-U/year increment [95\% CI: 0.07-0.83], $p=0.025$; HR 0.25 for every $10-\mathrm{U} /$ year increment [95\% CI: $0.08-0.82$ ], $p=0.022$ ).

\section{Discussion}

In the present study, we performed a combined analysis of data obtained in five longitudinal studies in Japanese patients with DM and revealed that three conventional measures of IMT (CCA-mean-IMT, CCA-max-IMT, and Max-IMT) were independent risk factors for the occurrence of CVD even after adjustment for conventional risk factors (Table 2). These findings are consistent with previous reports ${ }^{1,4-11)}$. Hazard ratios of CVD for these measures of IMT ranged from 1.07-1.12 (per 0.1-mm increment), suggesting that these parameters are useful as markers for CVD risk in daily clinical settings for DM care. In addition, time-dependent ROC curve analysis showed that the addition of CCA-mean-IMT, CCA-max-IMT or Max-IMT to the conventional risk factors only 
slightly but significantly improved the prediction ability for CVD (Table 3). However, the NRI statistics did not demonstrate a significant improvement in risk prediction. These results indicate that the addition of IMT to the conventional risk factors could bring about an improvement in the ability to predict CVD events in asymptomatic patients with DM,butthatits ; however, the extent of this improvement is smallas isthe case, similar to that in the general population ${ }^{1,12-15)}$. In subjects with metabolic syndrome or DM, Malik et al. assessed coronary artery calcium (CAC) and carotid IMT in the Multi-Ethnic Study of Atherosclerosis (MESA). They reported that the addition of IMT to the conventional risk factors could bring about an improvement in the ability to predict CVD, but that its extent is small, while CAC increased the C-statistic for CVD events over conventional risk factors and IMT $^{37)}$.

It is commonly accepted that lipid-rich plaques carry a higher risk for CVD events, since "disruption of a lipid-rich vulnerable plaque" could play a crucial role in the development of such events ${ }^{38,39)}$. Therefore, tissue characterization of a plaque lesion is considered to be useful for identifying patients at high risk for CVD. Interestingly, tissue characterization of carotid plaques can be estimated in a non-invasive manner using ultrasound, and echolucent plaques in the carotid artery was shown to correspond to lipid-rich atheromatous plaques ${ }^{40,41)}$. Notably, the current study showed that patients with low-GSM echolucent plaques in their carotid arteries had a significantly higher risk for cardiovascular events (Fig.1E). Furthermore, a multivariate Cox proportional hazards regression analysis revealed that plaque-GSM and the presence of low-GSM plaques were independent risk factors for the occurrence of CVD even after adjustment for conventional risk factors (HR 0.74 for every $10-\mathrm{U}$ increment [95\% CI: $0.62-0.90], p=0.002$ and HR 2.90 [95\% CI: 1.60-5.24], $p<0.001$, respectively; Table 2). These results were consistent with the concept that individuals who have echolucent, lipidrich, vulnerable plaques in their carotid arteries are prone to develop cardiovascular events. However, it did not increase the C-statistic when added to the best predictive models constructed for the present study subjects using nine conventional risk factors or the FRS. These results suggested that addition of carotid GSM to conventional risk factors may improve the predictive ability for CVD in asymptomatic patients with DM, but that the extent of this prediction would be small.

Theoretically, the progression of IMT reflects the progression of atherosclerosis, and thus could reflect the development of CVD events. Indeed, several pre- vious studies, including a meta-analysis, showed that a change over time in IMT (IMT change) predicted the future onset of CVD events and could, therefore, be used as a surrogate clinical endpoint for the development of $\mathrm{CVD}^{42-44)}$. However, recent meta-analyses in the general population as well as in subjects with DM contradicted this idea ${ }^{16,17)}$. Interestingly, the present study demonstrated that IMT change is a prognostic factor for CVD (Table 4). Although our finding is inconsistent with previous meta-analyses, there are several possible explanations for this discrepancy. First, different definitions of IMT in Western countries and in Japan might be a possible explanation. In Western countries, it is recommended that measurement of IMT should be performed in a region free of plaques ${ }^{45)}$ and the distinction between IMT and plaque should be made clearly. In contrast, the Japan Society of Ultrasonics in Medicine recommends that the measurement of IMT should be performed at the site of greatest thickness, including in plaque lesions ${ }^{31)}$. It is known that the process of IMT increase is a complex phenomenon, not determined by atherosclerotic risk factors alone ${ }^{46)}$, and that carotid plaques are a more sensitive and representative index of the atherosclerotic burden than IMT, with a greater predictive value for cardiovascular events ${ }^{47-49)}$. The progression of IMT including plaques rather than that excluding plaques might thus correlate more strongly with cardiovascular events. Second, random measurement errors at baseline and follow-up are accumulated into the index of IMT change. Therefore, the accuracy and reproducibility of the measurement of IMT is more important in the evaluation of the association between IMT change and CVD events as compared to those between baseline IMT and CVD events. A meta-analysis utilizing data from a number of institutions using different imaging modalities and different ultrasound protocols would tend to impair the accuracy and reproducibility in the measurement of IMT, and, thus, may have difficulty in demonstrating a significant association between IMT change and CVD events. On the other hand, our study used a unified ultrasound protocol, which could contribute to demonstrating the association between IMT change and CVD events.

Regarding GSM, there has been no evaluation of whether its change over time can be used as a therapeutic outcome. Interestingly, our study revealed that change over time in Mean-GSM was an independent risk factor for CVD events, even after adjustment for conventional risk factors, suggesting that Mean-GSM change can be a prognostic factor for CVD.

Several limitations of our study should be discussed. First, the numbers of the patients with data for 
Max-IMT $(n=540)$, Mean-GSM $(n=529)$, and plaque-GSM $(n=534)$ were small compared with the total of 3263 patients, which may have decreased the strength of the conclusion.

Second, the number of patients who developed CVD events during the observation period was relatively small, especially in the analyses that used MeanGSM and plaque-GSM. To conclusively demonstrate that the presence of echolucent carotid plaques is consistently and independently associated with CVD even after adjustment for conventional risk factors, further studies with large sample sizes would be necessary. The clinical utility of ultrasonic tissue characterization of carotid plaque as a stratification tool for CVD risk should be also confirmed in further studies.

Third, this study was performed in Japan and the IMT was measured at the site of greatest thickness, including plaque lesions according to the Japanese guidelines ${ }^{31}$. As a result, in many cases, the carotid "IMT (especially, Max-IMT)" measured in our study would represented carotid "plaque thickness" rather than carotid "IMT" measured in accordance with the Mannheim consensus (excluding plaques) in the Western countries ${ }^{45}$. Interestingly, it has been reported that plaque thickness, compared with IMT, can more accurately predict cardiovascular events ${ }^{4-49)}$. Therefore, it would not be appropriate to make a simple comparison between our findings on IMT and those of previous reports from Western countries.

Fourth, we adopted the FRS, the most famous risk score for CVD, as a typical CVD risk score based on conventional risk factors. However, there are several concerns for the application of the FRS to the Japanese population since there are non-negligible differences in the crude incidence of coronary artery disease, the ratio of stroke to coronary artery disease, and the risk profiles of CVD between Japanese and Americans. Indeed, a previous study revealed that the FRS overestimated the risk of coronary artery disease in the Japanese population ${ }^{50)}$. In addition, previous studies indicated that risk scores developed in the general population showed poorer performance when they were applied to patients with DM. Therefore, we might have adopted the JDCS/J-EDIT (JJ) risk engine, which had been developed for predicting macro- and microvascular complications in Japanese patients with type $2 \mathrm{DM}^{51)}$. However, we were not able to calculate the JJ risk engine due to lack of data on leisure-time physical activity.

Finally, recent studies indicate that it would be better to measure the carotid plaque burden as total plaque area ${ }^{52-54)}$ or volume ${ }^{55)}$, and that in patients without plaques it would be better to measure vessel wall volume ${ }^{56,57)}$ than IMT. However, the present study cannot address these points due to lack of data.

Notwithstanding these limitations, the present study suggests that the use of IMT-related measures, in addition to conventional risk factors or an established cardiovascular risk score, could bring about an improvement in the ability to predict CVD events in asymptomatic patients with DM, despite its small extent. It is also suggested that changes over time in carotid ultrasonography measures including IMT and GSM may be used as therapeutic outcome measures. In conclusion, our study indicates that measures derived from carotid ultrasonography can provide useful information for identifying patients with a high risk of CVD.

\section{Declarations}

\section{Ethics Approval and Consent to Participate}

The study protocol was approved by the Research Ethics Committee of the Osaka University Graduate School of Medicine, and the study was conducted in accordance with the principles of the Helsinki Declaration. This study is a retrospective analysis of the obtained data carried out by the opt-out method of our hospital website.

\section{Competing Interests}

None.

\section{Funding}

This research was supported by the Japan Agency for Medical Research and Development (AMED) [16ek0210061h000] and the Grants-in-Aid for Scientific Research from the Japanese Ministry of Education, Science, Sports, Culture and Technology [KAKENHI 16K09747].

\section{Authors' Contributions}

All authors contributed to the concept and design of this ongoing study. All authors read and approved the final manuscript. NK was the principal guarantor of this work, has full access to all the data, and takes responsibility for the integrity of the data and accuracy of its analysis.

\section{Acknowledgments}

The authors gratefully acknowledge the assistance of H Yamada and D Takayama (Soiken Holdings Inc., Tokyo, Japan) in performing the statistical analysis. 


\section{References}

1) Nezu T, Hosomi N, Aoki S, Matsumoto M: Carotid Intima-Media Thickness for Atherosclerosis. J Atheroscler Thromb, 2016; 23: 18-31

2) Huang LC, Lin RT, Chen CF, Chen $\mathrm{CH}$, Juo SH, Lin HF: Predictors of Carotid Intima-Media Thickness and Plaque Progression in a Chinese Population. J Atheroscler Thromb, 2016; 23: 940-949

3) Wada S, Koga M, Toyoda K, Minematsu K, Yasaka M, Nagai Y, Aoki S, Nezu T, Hosomi N, Kagimura T, Origasa H, Kamiyama K, Suzuki R, Ohtsuki T, Maruyama H, Kitagawa K, Uchiyama S, Matsumoto M; Japan Statin Treatment Against Recurrent Stroke (J-STARS) Echo Study Collaborators: Factors Associated with IntimaMedia Complex Thickness of the Common Carotid Artery in Japanese Noncardioembolic Stroke Patients with Hyperlipidemia: The J-STARS Echo Study. J Atheroscler Thromb, 2018; 25: 359-373

4) Nagai K, Shibata S, Akishita M, Sudoh N, Obara T, Toba K, Kozaki K: Efficacy of combined use of three non-invasive atherosclerosis tests to predict vascular events in the elderly; carotid intima-media thickness, flow-mediated dilation of brachial artery and pulse wave velocity. Atherosclerosis, 2013; 231: 365-370

5) Salonen JT, Salonen R: Ultrasonographically assessed carotid morphology and the risk of coronary heart disease. Atheroscler Thromb, 1991; 11: 1245-1249

6) Bots ML, Hoes AW, Koudstaal PJ, Hoffman A, Grobbee DE: Common carotid intima-media thickness and risk of stroke and myocardial infarction: the Rotterdam Study. Circulation, 1997; 96: 1432-1437

7) O'Leary DH, Polak JF, Kronmal RA, Manolio TA, Burke GL, Wolfson SK Jr.: Carotid-artery intima and media thickness as a risk factor for myocardial infarction and stroke in older adults. Cardiovascular Health Study Collaborative Research Group. N Engl J Med, 1999; 340: $14-22$

8) Lorenz MW, Markus HS, Bots ML, Rosvall M, Sitzer M: Prediction of clinical cardiovascular events with carotid intima-media thickness: a systematic review and metaanalysis. Circulation, 2007; 115: 459-467

9) Mukamal KJ, Kizer JR, Djoussé L, Ix JH, Zieman S, Siscovick DS, Sibley CT, Tracy RP, Arnold AM: Prediction and classification of cardiovascular disease risk in older adults with diabetes. Diabetologia, 2013; 56: 275-283

10) Novo S, Peritore A, Trovato RL, Guarneri FP, Di Lisi D, Muratori I, Novo G: Preclinical atherosclerosis and metabolic syndrome increase cardio- and cerebrovascular events rate: a 20-year follow up. Cardiovasc Diabetol, 2013; 12: 155. doi: 10.1186/1475-2840-12-155

11) Lau KK, Wong YK, Chan YH, Yiu KH, Teo KC, Li LS, Ho SL, Chan KH, Siu CW, Tse HF: Prognostic implications of surrogate markers of atherosclerosis in low to intermediate risk patients with type 2 diabetes. Cardiovasc Diabetol, 2012; 11: 101. doi: 10.1186/1475-2840-11101

12) Nambi V, Chambless L, Folsom AR, He M, Hu Y, Mosley T, Volcik K, Boerwinkle E, Ballantyne CM: Carotid intima-media thickness and presence or absence of plaque improves prediction of coronary heart disease risk: the
ARIC (Atherosclerosis Risk in Communities) study. J Am Coll Cardiol, 2010; 55: 1600-1607

13) Polak JF, Pencina MJ, Pencina KM, O’Donnell CJ, Wolf PA, D'Agostino RB Sr.: Carotid-wall intima-media thickness and cardiovascular events. N Engl J Med, 2011; 365: 213-221

14) Baldassarre D, Hamsten A, Veglia F, de Faire U, Humphries SE, Smit AJ, Giral P, Kurl S, Rauramaa R, Mannarino E, Grossi E, Paoletti R, Tremoli E; IMPROVE Study Group: Measurements of carotid intima-media thickness and of interadventitia common carotid diameter improve prediction of cardiovascular events: results of the IMPROVE (Carotid Intima Media Thickness [IMT] and IMT-Progression as Predictors of Vascular Events in a High Risk European Population) study. J Am Coll Cardiol, 2012; 60: 1489-1499

15) Yeboah J, McClelland RL, Polonsky TS, Burke GL, Sibley CT, O'Leary D, Carr JJ, Goff DC, Greenland P, Herrington DM: Comparison of novel risk markers for improvement in cardiovascular risk assessment in intermediate-risk individuals. JAMA, 2012; 308: 788-795

16) Lorenz MW, Polak JF, Kavousi M, Mathiesen EB, Völzke H, Tuomainen TP, Sander D, Plichart M, Catapano AL, Robertson CM, Kiechl S, Rundek T, Desvarieux M, Lind L, Schmid C, DasMahapatra P, Gao L, Ziegelbauer K, Bots ML, Thompson SG; PROG-IMT Study Group: Carotid intima-media thickness progression to predict cardiovascular events in the general population (the PROG-IMT collaborative project): a meta-analysis of individual participant data. Lancet, 2012; 379(9831): 2053-2062

17) Lorenz MW, Price JF, Robertson C, Bots ML, Polak JF, Poppert H, Kavousi M, Dörr M, Stensland E, Ducimetiere P, Ronkainen K, Kiechl S, Sitzer M, Rundek T, Lind L, Liu J, Bergström G, Grigore L, Bokemark L, Friera A, Yanez D, Bickel H, Ikram MA, Völzke H, Johnsen SH, Empana JP, Tuomainen TP, Willeit P, Steinmetz H, Desvarieux M, Xie W, Schmidt C, Norata GD, Suarez C, Sander D, Hofman A, Schminke U, Mathiesen E, Plichart M, Kauhanen J, Willeit J, Sacco RL, McLachlan S, Zhao D, Fagerberg B, Catapano AL, Gabriel R, Franco OH, Bülbül A, Scheckenbach F, Pflug A, Gao L, Thompson SG: Carotid Intima Media Thickness Progression and Risk of Vascular Events in People With Diabetes: Results From the PROG-IMT Collaboration. Diabetes Care, 2015; 38: 1921-1929

18) Takiuchi S, Rakugi H, Honda K, Masuyama T, Hirata N, Ito H, Sugimoto K, Yanagitani Y, Moriguchi K, Okamura A, Higaki J, Ogihara T: Quantitative ultrasonic tissue characterization can identify high-risk atherosclerotic alteration in human carotid arteries. Circulation, 2000; 102: 766-770

19) Falkowski A, Kaczmarczyk M, Cieszanowski A, Goracy I, Poncyliusz W, Wilk G: Computer-assisted characterisation of a carotid plaque. Med Sci Monit, 2004; 10: 67-70

20) Nicolaides AN, Kakkos SK, Kyriacou E, Griffin M, Sabetai M, Thomas DJ, Tegos T, Geroulakos G, Labropoulos N, Doré CJ, Morris TP, Naylor R, Abbott AL; Asymptomatic Carotid Stenosis and Risk of Stroke (ACSRS) Study Group: Asymptomatic internal carotid artery stenosis and cerebrovascular risk stratification. J Vasc Surg, 
2010; 52: 1486-1496. e1-5

21) Katakami N, Takahara M, Kaneto H, Sakamoto K, Yoshiuchi K, Irie Y, Kubo F, Katsura T, Yamasaki Y, Kosugi K, Shimomura I: Ultrasonic tissue characterization of carotid plaque improves the prediction of cardiovascular events in diabetic patients: a pilot study. Diabetes Care, 2012; 35: 2640-2646

22) Irie $Y$, Katakami $N$, Kaneto $H$, Takahara $M$, Nishio $M$, Kasami R, Sakamoto K, Umayahara Y, Sumitsuji S, Ueda Y, Kosugi K, Shimomura I: The utility of ultrasonic tissue characterization of carotid plaque in the prediction of cardiovascular events in diabetic patients. Atherosclerosis, 2013; 230: 399-405

23) Yoshida M, Mita T, Yamamoto R, Shimizu T, Ikeda F, Ohmura C, Kanazawa, Hirose T, Kawamori R, Watada H: Combination of the Framingham risk score and carotid intima-media thickness improves the prediction of cardiovascular events in patients with type 2 diabetes. Diabetes Care, 2012; 35: 178-180

24) Katakami N, Mita T, Yoshii H, Onuma T, Kaneto $H$, Osonoi T, Shiraiwa T, Kosugi K, Umayahara Y, Yamamoto $\mathrm{T}$, Yokoyama $\mathrm{H}$, Kuribayashi N, Jinnouchi $\mathrm{H}$, Gosho M, Watada H, Shimomura I; Collaborators on the Study of Preventive Effects of Alogliptin on Diabetic Atherosclerosis (SPEAD-A) Trial: Rationale, design, and baseline characteristics of a trial for prevention of diabetic atherosclerosis using a DPP-4 inhibitor: the Study of Preventive Effects of Alogliptin on Diabetic Atherosclerosis (SPEAD-A). J Atheroscler Thromb, 2013; 20: 893-902

25) Mita M, Katakami N, Yoshii H, Onuma T, Kaneto H, Osonoi T, Shiraiwa T, Kosugi K, Umayahara Y, Yamamoto T, Yokoyama H, Kuribayashi N, Jinnouchi $H$, Gosho M, Shimomura I, Watada H; Collaborators on the Study of Preventive Effects of Alogliptin on Diabetic Atherosclerosis (SPEAD-A) Trial: Alogliptin, a dipeptidyl peptidase- 4 inhibitor, prevents the progression of carotid atherosclerosis in patients with type 2 diabetes mellitus: The Study of Preventive Effects of Alogliptin on Diabetic Atherosclerosis (SPEAD-A). Diabetes Care, 2016; 39: 139-148

26) Mita T, Katakami N, Shiraiwa T, Yoshii H, Onuma T, Kuribayashi N, Osonoi T, Kaneto H, Kosugi K, Umayahara Y, Yamamoto T, Matsumoto K, Yokoyama H, Tsugawa M, Gosho M, Shimomura I, Watada H: Rationale, design, and baseline characteristics of a clinical trial for prevention of atherosclerosis in patients with insulintreated type 2 diabetes mellitus using DPP-4 inhibitor: The Sitagliptin Preventive study of Intima-media thickness Evaluation (SPIKE). Diabetol Metab Syndr, 2014; 6: 35. doi: 10.1186/1758-5996-6-35

27) Mita M, Katakami N, Shiraiwa T, Yoshii H, Onuma T, Kuribayashi N, Osonoi T, Kaneto H, Kosugi K, Umayahara U, Yamamoto T, Matsumoto K, Yokoyama H, Tsugawa M, Gosho M, Shimomura I, Watada H, and Collaborators on Sitagliptin Preventive study of Intima media thickness Evaluation (SPIKE) Trial: Sitagliptin attenuates the progression of carotid intima-media thickening in insulin-treated patients with type 2 diabetes mellitus: The Sitagliptin Preventive study of Intima-media thickness Evaluation (SPIKE): a randomized controlled trial. Diabetes Care, 2016; 39: 455-464
28) The Japanese Circulation Joint Working Group (20002001): Guidelines for management of acute coronary syndrome without persistent ST segment elevation (JCS 2002). Circulation Journal, 2002; 66: Suppl. IV

29) The Japanese Circulation Joint Working Group (2004): Guidelines for diagnostic evaluation of patients with chronic ischemic heart disease (JCS 2005). 2005 [http:// www.j-circ.or.jp/guideline/pdf/JCS2005_yokoyama_ h.pdf]

30) Special report from the National Institute of Neurological Disorders and Stroke. Classification of cerebrovascular diseases III. Stroke, 1990; 21: 637-676

31) Terminology and Diagnostic Criteria Committee, Japan Society of Ultrasonics in Medicine: Subcommittee for Preparing Guidelines for Ultrasound Diagnosis of Carotid Artery: Standard method for ultrasound evaluation of carotid artery lesions. Jpn J Med Ultrasonics, 2009; 36: 501-518

32) Sabetai MM, Tegos TJ, Nicolaides AN, Dhanjil S, Pare GJ, Stevens JM: Reproducibility of computer-quantified carotid plaque echogenicity: can we overcome the subjectivity? Stroke, 2000; 31: 2189-2196

33) D'Agostino RB Sr, Vasan RS, Pencina MJ, Wolf PA, Cobain M, Massaro JM, Kannel WB: General cardiovascular risk profile for use in primary care: the Framingham Heart Study. Circulation, 2008; 117: 743-753

34) Japan Diabetes Society: Diabetes mellitus treatment guideline based on scientific ground (revised edition 2). Nankodo: Japan Diabetes Society, 2007; 257-272

35) Heagerty PJ, Lumley T, Pepe MS: Time-dependent ROC curves for censored survival data and a diagnostic marker. Biometrics, 2000; 56: 337-344

36) Steyerberg, EW, Pencina MJ: Reclassification calculations for persons with incomplete follow-up. Annals of Internal Medicine, 2010; 152: 195-196

37) Malik S, Budoff MJ, Katz R, Blumenthal RS, Bertoni AG, Nasir K, Szklo M, Barr RG, Wong ND: Impact of subclinical atherosclerosis on cardiovascular disease events in individuals with metabolic syndrome and diabetes: the multi-ethnic study of atherosclerosis. Diabetes Care, 2011; 34: 2285-2290

38) Davies MJ, Richardson PD, Woolf N, Katz DR, Mann J: Risk of thrombosis in human atherosclerotic plaques: role of extracellular lipid, macrophage, and smooth muscle cell content. Br Heart J, 1993; 69: 377-381

39) Falk E: Pathogenesis of atherosclerosis. J Am Coll Cardiol, 2006; 47: C7-12

40) Grønholdt ML, Wiebe BM, Laursen H, Nielsen TG, Schroeder TV, Sillesen H: Lipid-rich carotid artery plaques appear echolucent on ultrasound B-mode images and may be associated with intraplaque haemorrhage. Eur J Vasc Endovasc Surg, 1997; 14: 439-445

41) Lal BK, Hobson RW 2nd, Pappas PJ, Kubicka R, Hameed M, Chakhtoura EY, Jamil Z, Padberg FT Jr, Haser PB, Durán WN: Pixel distribution analysis of B-mode ultrasound scan images predicts histologic features of atherosclerotic carotid plaques. J Vasc Surg, 2002; 35: $1210-1217$

42) Hodis HN, Mack WJ, LaBree L, Selzer RH, Liu CR, Liu $\mathrm{CH}$, Azen SP: The role of carotid arterial intima-media thickness in predicting clinical coronary events. Ann 
Intern Med, 1998; 128: 262-269

43) Goldberger ZD, Valle JA, Dandekar VK, Chan PS, Ko DT, Nallamothu BK: Are changes in carotid intimamedia thickness related to risk of nonfatal myocardial infarction? A critical review and meta-regression analysis. Am Heart J, 2010; 160: 701-714

44) Okayama KI, Mita T, Gosho M, Yamamoto R, Yoshida M, Kanazawa A, Kawamori R, Fujitani Y, Watada H: Carotid intima-media thickness progression predicts cardiovascular events in Japanese patients with type 2 diabetes. Diabetes Res Clin Pract, 2013; 101: 286-292

45) Touboul PJ, Hennerici MG, Meairs S, Adams H, Amarenco P, Bornstein N, Csiba L, Desvarieux M, Ebrahim S, Fatar M, Hernandez R, Jaff M, Kownator S, Prati P, Rundek T, Sitzer M, Schminke U, Tardif JC, Taylor A, Vicaut E, Woo KS, Zannad F, Zureik M: Mannheim Carotid Intima-Media Thickness Consensus (20042006). Cerebrovasc Dis, 2007; 23: 75-80

46) Bortel L: What does intima-media thickness tell us? J Hypertens, 2005; 23: 37-39

47) Barnett PA, Spence JD, Manuck SB, Jennings JR: Psychological stress and the progression of carotid artery disease. J Hypertens, 1997; 15: 49-55

48) Spence JD, Eliasziw M, DiCicco M, Hackam DG, Galil R, Lohmann T: Carotid plaque area: a tool for targeting and evaluating vascular preventive therapy. Stroke, 2002; 33: 2916-2922

49) Inaba Y, Chen JA, Bergmann SR: Carotid plaque, compared with carotid intima-media thickness, more accurately predicts coronary artery disease events: A metaanalysis. Atherosclerosis, 2011; 220: 128-133

50) Nishimura K, Okamura T, Watanabe M, Nakai M, Takegami M, Higashiyama A, Kokubo Y, Okayama A, Miyamoto Y: Predicting coronary heart disease using risk factor categories for a Japanese urban population, and comparison with the framingham risk score: the suita study. J Atheroscler Thromb, 2014; 21: 784-798

51) Tanaka $S$, Tanaka $S$, Iimuro $S$, Yamashita H, Katayama S,
Akanuma Y, Yamada N, Araki A, Ito H, Sone H, Ohashi Y; Japan Diabetes Complications Study Group; Japanese Elderly Diabetes Intervention Trial Group: Predicting macro- and microvascular complications in type 2 diabetes: the Japan Diabetes Complications Study/the Japanese Elderly Diabetes Intervention Trial risk engine. Diabetes Care, 2013; 36: 1193-1199

52) Spence JD, Eliasziw M, DiCicco M, Hackam DG, Galil R, Lohmann T: Carotid plaque area: a tool for targeting and evaluating vascular preventive therapy. Stroke, 2002; 33: 2916-2922

53) Johnsen SH, Mathiesen EB, Joakimsen O, Stensland E, Wilsgaard T, Løchen ML, Njølstad I, Arnesen E: Carotid atherosclerosis is a stronger predictor of myocardial infarction in women than in men: a 6-year follow-up study of 6226 persons: the Tromso Study. Stroke, 2007; 38: 28732880

54) Mathiesen EB, Johnsen SH, Wilsgaard T, Bonaa KH, Lochen ML, Njolstad I: Carotid Plaque Area and IntimaMedia Thickness in Prediction of First-Ever Ischemic Stroke: A 10-Year Follow-Up of 6584 Men and Women: The Tromso Study. Stroke, 2011; 42: 972-978

55) Baber U, Mehran R, Sartori S, Schoos MM, Sillesen H, Muntendam P, Garcia MJ, Gregson J, Pocock S, Falk E, Fuster V: Prevalence, impact, and predictive value of detecting subclinical coronary and carotid atherosclerosis in asymptomatic adults: the BioImage study. J Am Coll Cardiol, 2015; 65: 1065-1074

56) Egger M, Spence JD, Fenster A, Parraga G: Validation of $3 \mathrm{~d}$ ultrasound vessel wall volume: an imaging phenotype of carotid atherosclerosis. Ultrasound Med Biol, 2007; 33: 905-914

57) Shai I, Spence JD, Schwarzfuchs D, Henkin Y, Parraga G, Rudich A, Fenster A, Mallett C, Liel-Cohen N, Tirosh A, Bolotin A, Thiery J, Fiedler GM, Blüher M, Stumvoll M, Stampfer MJ; DIRECT Group: Dietary intervention to reverse carotid atherosclerosis. Circulation, 2010; 121: $1200-1228$ 
Supplemental Table 1. List of the longitudinal studies from which data were utilized to construct the combined dataset

(1) "The development of tailor-made treatment for diabetic complications using DNA chips," an observational study that was conducted at Osaka University Hospital and Naka-Kinen Hospital from January 11, 2005, to March 24, 2015.

(2) "Predictive value of carotid intima-media thickness for cardiovascular disease in type 2 diabetic patients," an ongoing observational study that started in 2003 at Juntendo University Hospital [Ref. 20].

(3) "Clinical research on identifying patients at high risk for severe coronary disease among those with asymptomatic type 2 diabetes mellitus without a history of coronary artery disease," an ongoing observational study that started in 2007 at Osaka Police Hospital.

(4) SPEAD-A Study, a clinical study started at Juntendo University Hospital and other institutions in 2011 [Ref. 21, 22], and its extension study called "SPEAD-A Extension Study".

(5) SPIKE Study, a clinical study started in Juntendo University Hospital and other institutions in 2012 [Ref. 23, 24], and its extension study called the "SPIKE Extension Study".

\begin{tabular}{|c|c|c|c|c|c|c|}
\hline Study & Primary aim & Major eligibility criteria & $\begin{array}{c}\text { Total number } \\
\text { of subjects }\end{array}$ & $\begin{array}{l}\text { Follow-up } \\
\text { period }\end{array}$ & $\begin{array}{c}\text { Age } \\
(\text { mean } \pm S D)\end{array}$ & $\begin{array}{c}\text { Sex (proportion } \\
\text { of men, \%) }\end{array}$ \\
\hline (1) & $\begin{array}{l}\text { To develop tailor-made treatment for } \\
\text { diabetic complications through the } \\
\text { identification of their genetic and } \\
\text { environmental risk factors }\end{array}$ & $\begin{array}{l}\text { (1) patients with DM } \\
\text { (2) free of severe PAD or cerebral } \\
\text { infarction sequelae } \\
\text { (3) age } \geq 20 \text { but }<76 \text { years, } \\
\text { irrespective of sex }\end{array}$ & 2300 & $2005-2015$ & $59.8 \pm 10.6$ & 64.3 \\
\hline (2) & $\begin{array}{l}\text { To evaluate whether carotid IMT add } \\
\text { value to the Framingham risk score in } \\
\text { predicting the development of } \\
\text { cardiovascular diseases }\end{array}$ & $\begin{array}{l}\text { (1) patients with T2DM } \\
\text { (2) free of a history of } \\
\text { cardiovascular disease } \\
\text { (3) age } \geq 30 \text { but }<76 \text { years, } \\
\text { irrespective of sex }\end{array}$ & 783 & $\begin{array}{c}2003 \text { - } \\
\text { ongoing }\end{array}$ & $58.2 \pm 9.6$ & 66.2 \\
\hline (3) & $\begin{array}{l}\text { To evaluate the association between } \\
\text { carotid IMT and the presence of } \\
\text { severe atherosclerotic lesions in } \\
\text { coronary arteries as well as future } \\
\text { onset of cardiovascular disease }\end{array}$ & $\begin{array}{l}\text { (1) patients with T2DM } \\
\text { (2) free of a history of coronary } \\
\text { artery disease }\end{array}$ & 333 & $\begin{array}{c}2007 \text { - } \\
\text { ongoing }\end{array}$ & $64.6 \pm 7.6$ & 70.9 \\
\hline (4) & $\begin{array}{l}\text { To investigate the effects of alogliptin, } \\
\text { a DPP- } 4 \text { inhibitor, on the progression } \\
\text { of carotid atherosclerosis in patients } \\
\text { with type } 2 \text { diabetes mellitus. }\end{array}$ & $\begin{array}{l}\text { (1) patients with T2DM } \\
\text { (2) free of a history of apparent } \\
\text { cardiovascular diseases } \\
\text { (3) age } \geq 30 \text { years, irrespective of sex }\end{array}$ & 322 & $\begin{array}{c}2011 \text { - } \\
\text { ongoing }\end{array}$ & $64.6 \pm 9.4$ & 61.8 \\
\hline (5) & $\begin{array}{l}\text { To assess the effects of sitagliptin, a } \\
\text { dipeptidyl peptidase } 4 \text { inhibitor, on } \\
\text { carotid intima-media thickness in type } \\
2 \text { diabetes mellitus. }\end{array}$ & $\begin{array}{l}\text { (1) patients with T2DM using insulin } \\
\text { (2) free of a history of apparent } \\
\text { cardiovascular diseases } \\
\text { (3) age } \geq 30 \text { but }<80 \text { years, } \\
\text { irrespective of sex }\end{array}$ & 274 & $\begin{array}{l}2012 \text { - } \\
\text { ongoing }\end{array}$ & $63.7 \pm 9.8$ & 60.2 \\
\hline
\end{tabular}

